\title{
KERNEL-BASED NOISE FILTERING OF NEUTRON DETECTOR SIGNALS
}

\author{
MOON-GHU PARK*, HO-CHEOL SHIN and EUN-KI LEE \\ Korea Electric Power Research Institute \\ 103-16 Munji-Dong, Yusung-Gu, Daejeon, Korea, 305-380 \\ "Corresponding author. E-mail : mgpark@kepri.re.kr
}

Received June 26, 2007

Accepted for Publication Septebmer 19, 2007

This paper describes recently developed techniques for effective filtering of neutron detector signal noise. In this paper, three kinds of noise filters are proposed and their performance is demonstrated for the estimation of reactivity. The tested filters are based on the unilateral kernel filter, unilateral kernel filter with adaptive bandwidth and bilateral filter to show their effectiveness in edge preservation. Filtering performance is compared with conventional low-pass and wavelet filters. The bilateral filter shows a remarkable improvement compared with unilateral kernel and wavelet filters. The effectiveness and simplicity of the unilateral kernel filter with adaptive bandwidth is also demonstrated by applying it to the reactivity measurement performed during reactor start-up physics tests.

KEYWORDS : Kernel, Wavelet, Noise, Filter, Reactivity

\section{INTRODUCTION}

Nuclear reactor power deviation from the critical state is a parameter of specific interest defined by the reactivity-measuring neutron population. Reactivity is an important quantity used to define many reactor startup physics parameters. [1] Time-dependent reactivity is normally determined by solving the inverse neutron kinetics equation with a reactivity computer to provide an on-line solution [2]. The neutron density measurement signal is normally noise-corrupted and the movement of control rods typically gives reactivity variation with edge signals like saw teeth. Those edge regions should be precisely preserved since the measured signal is used to estimate reactivity worth which is a crucial parameter for assuring the safety of nuclear reactors.

In this paper, three kinds of edge-preserving noise filters are proposed and their performance is demonstrated using stepwise signals. The tested filters are based on the unilateral kernel filter, unilateral kernel filter with adaptive bandwidth and bilateral filter to show their effectiveness in edge preservation. The bilateral filter shows a remarkable improvement compared with unilateral kernel and wavelet filters.

Section 2 summarizes the digital reactivity calculation method applied to the Direct Digital Reactivity Computer System (DDRCS ${ }^{\mathrm{TM}}$ ) which is based on inverse kinetics.

Section 3 describes the general framework of kernel regression filtering methods considering the various options to scale kernel bandwidth.

Section 4 gives the results of application and demonstration of the kernel filters using reactor physics test data for reactivity worth measurement and a simulated stepwise signal for edge preservation, respectively.

\section{REACTIVITY CALCULATION}

Reactivity measurement is normally performed by the inverse kinetics method based on the point reactor kinetics equation. It includes the external neutron source term, prompt and delayed neutron fractions [2, 3]. Using the inverse point kinetics equation, the measured reactivity is calculated by

$$
\rho(t)=\sum_{k} \beta_{k} \int_{-\infty}^{t} \frac{N^{\prime}\left(t^{\prime}\right)}{N(t)} e^{-\lambda_{k}\left(t-t^{\prime}\right)} d t^{\prime}+\Lambda \frac{N^{\prime}(t)}{N(t)}-\frac{s_{e x t}(t)}{N(t)}
$$

where, $\rho(t)$ : total core reactivity

$\beta_{k}: \mathrm{k}$-th group delayed neutron fraction

$N(t)$ : core-averaged neutron density

$A$ : prompt neutron generation time

$\lambda_{k}:$ k-th group delayed neutron decay constant

$S_{\text {ext }}$ : external source strength 
Equation (1) can be reduced easily from the traditional point kinetics equation. The first integral term of the right-hand side represents the precursor term. By using the traditional assumption about neutron density, which varies exponentially with time, the numerical solution of Equation (1) becomes

$$
\rho\left(t_{n}\right)=\sum_{k} \beta_{k}\left(e^{-\left(\lambda_{k}+\omega_{n}\right) \Delta_{n}} B_{n-1, k}+A_{n, k}\right)+\Lambda \omega_{n}-s_{e x i}(t) \frac{e^{-\left(\omega_{n} \Delta t_{n}\right.}}{N_{n-1}}
$$

where,

$$
A_{n, k}=\frac{\omega_{n}}{\lambda_{k}+\omega_{n}}\left(1-e^{-\left(\lambda_{k}+\omega_{n}\right) \Delta t_{n}}\right) \text { and } \omega_{n}=\ln \left(\frac{N_{n}}{N_{n-1}}\right) / \Delta t
$$

Figure 1. shows a schematic description of a digital reactivity measurement system including a noise filter for ex-core detector measurement.

\section{KERNEL REGRESSION FILTER}

Kernel regression is an old method for smoothing data; still, new work with this method continues at a rapid pace.
Traditional digital filters have their own dynamics and it is not easy to determine filter gains. A kernel regression filter is a kind of static filter and it is easy to get the desired performance with a simple adaptation of its variance. Kernel regression of statistics was derived independently by Nadaraya[4] and Moore[5]. Kernel regression is the estimation of the functional relationship $y(t)$ between two variables $y$ and $t$. Measurement produces a set of random variables $\left\{t_{i}, y_{i} ; i=1,2, \ldots, N\right\}$ on the interval $\left\{0 \leq t_{i} \leq T\right\}$. It is assumed that

$$
y_{i}=y\left(t_{i}\right)+\varepsilon
$$

where $\varepsilon$ is a random noise variable with the mean equal to zero. Since the purpose of the kernel regression filter in this paper is to smooth out the small local details and preserve edge signals, no specific noise characteristic of $\varepsilon$ is assumed.

The Nadaraya-Watson kernel regression estimate of $y(t)$ at $t=\tau$ from this random data is defined as the estimator $y(\tau)$ as

$$
\hat{y}(\tau)=\frac{\sum_{i=1}^{N} y_{i} k\left(\tau-t_{i}\right)}{\sum_{i=1}^{N} k\left(\tau-t_{i}\right)} .
$$

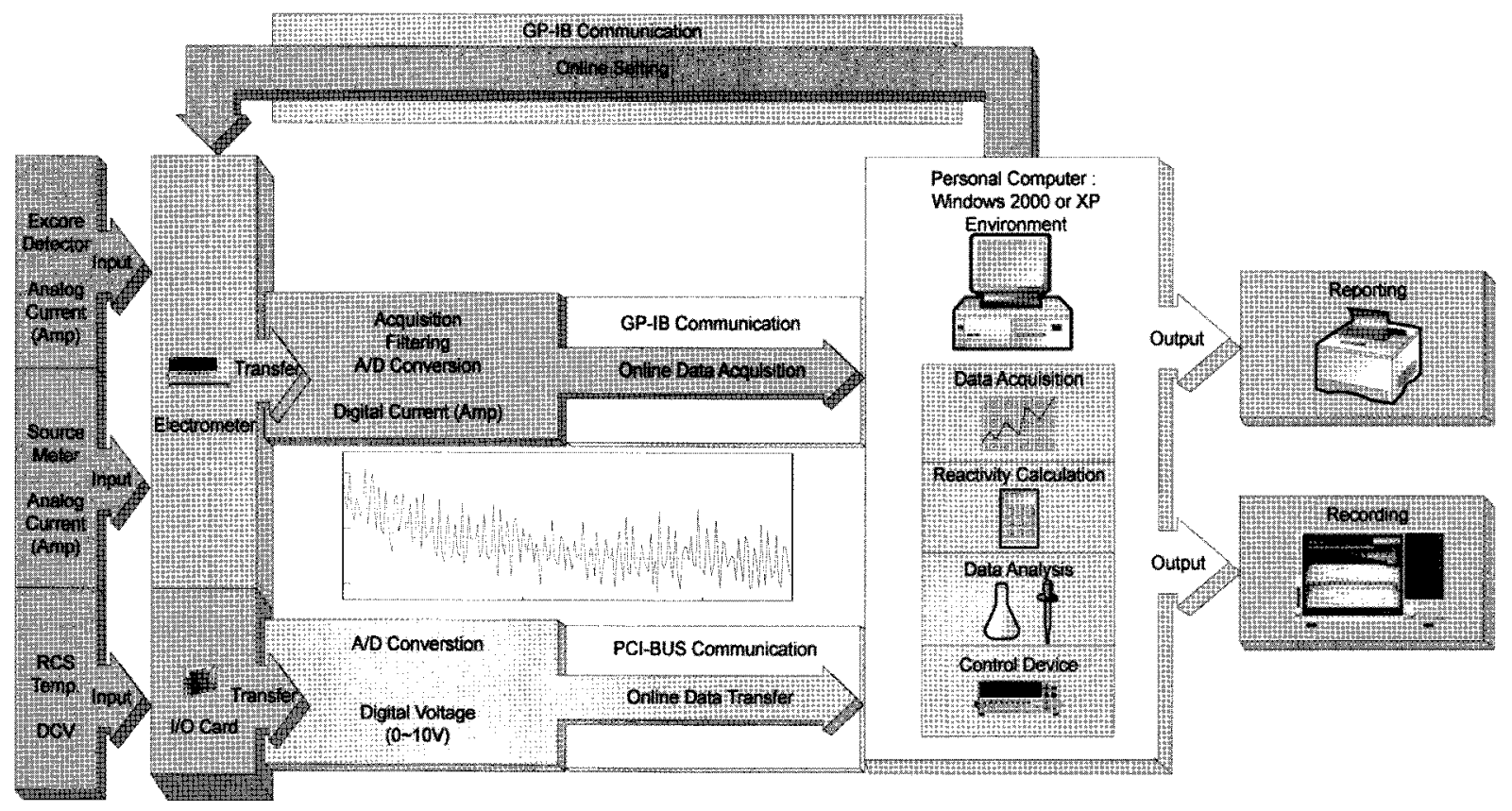

Fig. 1. Schematic Description of Digital Reactivity Measurement System 
The function $k\left(\tau-t_{i}\right)$ is the kernel function which can be chosen from a wide variety of symmetric functions.

\subsection{Kernel Regression Filter with Fixed Bandwidth}

In this paper, the Gaussian density function is used for the kernel function, i.e.,

$$
k(t)=\exp \left(-D\left(t_{i}, t_{q}\right)^{2} / \sigma^{2}\right)
$$

where $D$ is the distance metric and Euclidean distance is used here defined by

$$
D\left(t_{i}, t_{q}\right)=\left\|t_{i}-t_{q}\right\|=\sqrt{\left(t_{i}-t_{q}\right)^{2}}
$$

$t_{q}$ is the query point where the smoothed signal is to be generated in the interval of time series data $\left\{0 \leq t_{i} \leq T\right\} . \sigma^{2}$ is the bandwidth of the kernel which controls how widely the influencing measurements are spread around a query point. Bandwidth can also control the smoothness or roughness of a density estimate. Increasing the kernel width $\sigma^{2}$ means more distant points get an opportunity to influence the query point. As $\sigma^{2} \rightarrow \infty$, the smoothed signal tends to the global average. [6]

Figure 2 illustrates the filtering performance of a kernel filter as it relates to bandwidth size. For $\sigma^{2}=0.1$, the raw signal is perfectly reconstructed and for the large bandwidth $\sigma^{2}=100$, of , the filtered signal approaches the average value of the signal. Gaussian function is selected as the kernel function since it is simple and the only

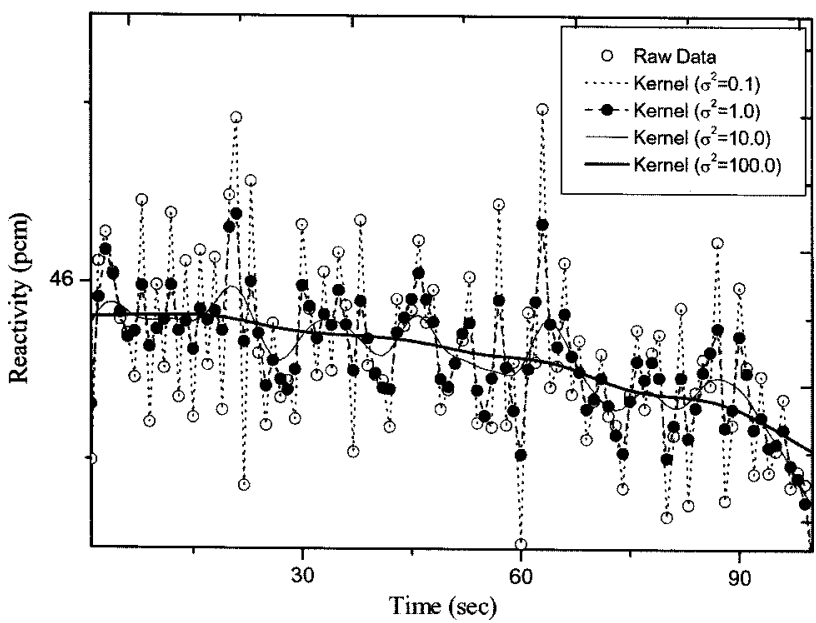

Fig. 2. Filtering Performance of Kernel Filter Depending on Bandwidth Size radial basis function which is factorisable.The significance of factorisability is that the synthesis of radial basis functions in many dimensions may be easier if they are factorisable. This property is especially attractive from an implementational point of view, as it offers the prospect of reduced programming complexity parallel and/or hardware implementation and so forth. The choice of kernel function is not critical for the noise filtering application but rather for the choice of bandwidth.

\subsection{Kernel Regression Filter with Adaptive Bandwidth}

In this section an adaptation algorithm is introduced to modify the bandwidth which can be reduced appropriately to preserve the edge signal as an inverse of the local distance as follows:

$$
\sigma(\tau)=\frac{1}{\sum_{i=1}^{N}\left(x(\tau)-x\left(t_{i}\right)\right)^{2}}
$$

where $N$ is the number of samples around the query points.

\subsection{Bilateral Kernel Filter}

The bilateral filter is a technique proposed by Tomasi and Manduchi [6]. This technique preserves edges by mixing a moving average technique with a nonlinear system of weights. It relies on an assumption that any noise is more uniformly distributed, and that signals have distinct edges or steps that can be detected by examining local differences. Each neighboring value is weighted on its proximity in space or time (a domain weight). A second weighting factor gives some measurement of local difference (a range weight). Often, these two weights are expressed as a pair of Gaussian distributions in a summation. In the time domain, this is expressed as:

$$
\begin{aligned}
k(t) & =k_{D}(\text { distance }) \times k_{G}(\text { feature }) \\
& =\exp \left(-D\left(t_{i}, t_{q}\right)^{2} / \sigma_{t}^{2}\right) \times \exp \left(-D\left(y_{i}, y_{q}\right)^{2} / \sigma_{x}^{2}\right)
\end{aligned}
$$

where $\sigma_{t}{ }^{2}, \sigma_{x}{ }^{2}$ are the variances of the spatial distances for noise rejection and feature preservation, respectively. The weighting factor plays a role with local difference information for edge preservation. Figure 3 shows the effect of the distance and feature weights for the bilateral filter. The weight for distance $k_{D}$ averages local small details and the weight for feature $k_{G}$ preserves edges of steps or large scale amps. The term $D\left(y_{i}, y_{q}\right)$ becomes large at the step change of $y_{q-1}$ and $y_{q}$ and this gives the same effect of decrease in bandwidth $\sigma_{x}^{2}$ which keeps the 


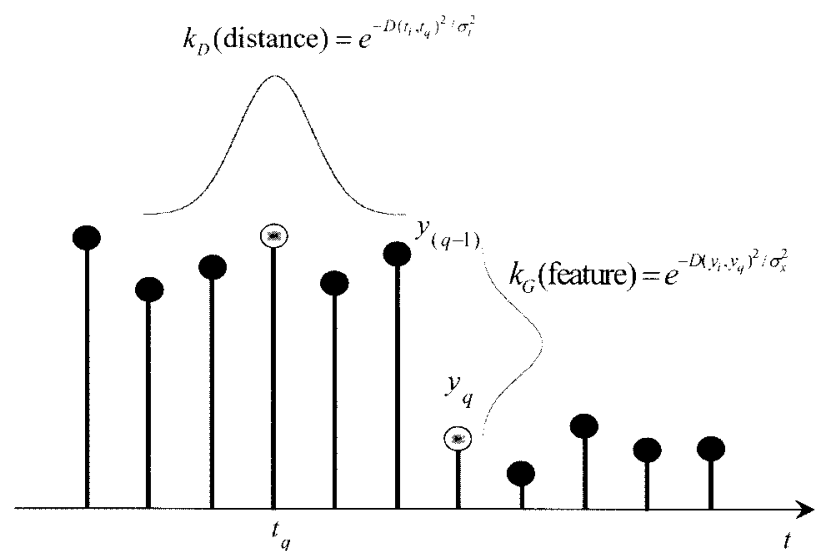

Fig. 3. Role of the Distance and Feature Weights for Bilateral Filter

high frequency components. This characteristic is an inherent feature of the bilateral filter.

\subsection{Wavelet De-Noising}

Wavelet is a waveform of effectively limited duration that has an average value of zero, which can be applied to non-stationary and transient signals. Wavelet analysis is the breaking up of a signal into shifted and scaled versions of the original (or mother) wavelet. Wavelet theory is one of the prevailing applications in the field of noise filtering. In this paper, the Coiflet is selected and the corresponding expansion coefficients can be found in [8] and the application to reactivity estimation is described in [9].

\section{APPLICATION AND DEMONSTRATION}

\subsection{Smooth Signal Filtering}

The measurement and validation of control rod bank (group) worths are typically required by the start-up physics test standard programs for Pressurized Water Reactors (PWR). Recently, the DCRM ${ }^{\mathrm{TM}}$ method was developed and implemented by KEPRI (Korea Electric Power Research Institute). The method is based on a fast and complete bank insertion within a short period of time which makes the range of the reactivity variation very large, from below the background gamma level to the vicinity of the nuclear heating point. [2] The weak flux signal below the background gamma level is highly noise-contaminated which invokes a large reactivity fluctuation. This paper describes an efficient noise filtering method with the unilateral kernel filter for that very weak but smooth signal. The performance of the developed method is demonstrated with the measurement data at a Korea Standard Nuclear Plant. During that test, the reactivity measurements were performed for control banks. Figure 4 shows the typical variation of the flux signal during the DCRM ${ }^{\mathrm{TM}}$ test which is the most severely noise-contaminated case. Figure 4 shows the effective and smooth kernel-filtered result for the weak neutron flux signal below the gamma background.

Figure 5 gives the control bank reactivity calculated with a noisy neutron flux signal and a filtered signal. After the bank insertion, it can be seen that the noise components are dominant when the control bank is in the core bottom region where only sub-critical multiplication exists. Since the reactivity calculation is very sensitive to variation in neutron flux, a noisy signal should be appropriately filtered. Figure 5 clearly shows the effective noise filtering in the reactivity calculation. Thus the kernel-based filter is proved to be applicable to estimate the reactivity variation during a DCRM ${ }^{\mathrm{TM}}$ test.

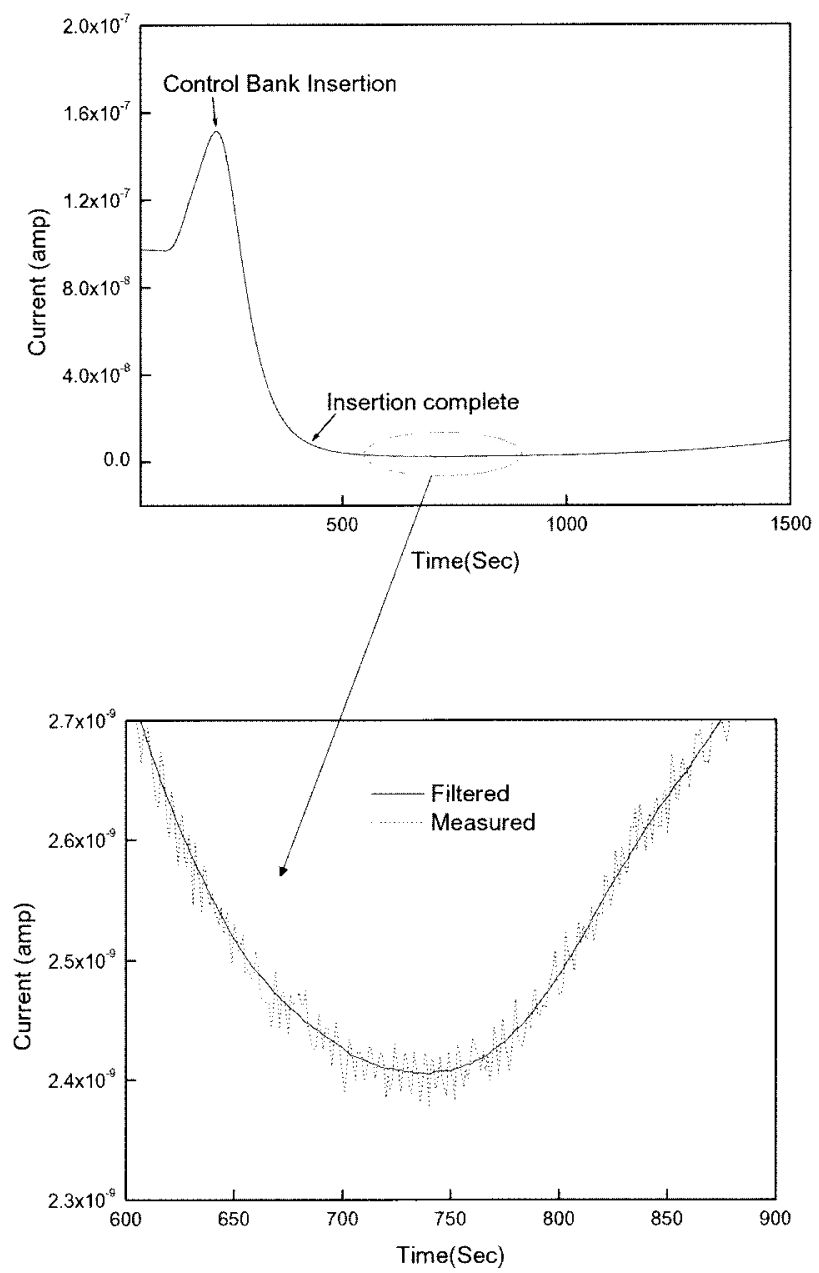

Fig. 4. Typical Variation of the Neutron Flux Signal During DCRM $^{\mathrm{TM}}$ Test at a KSNP 


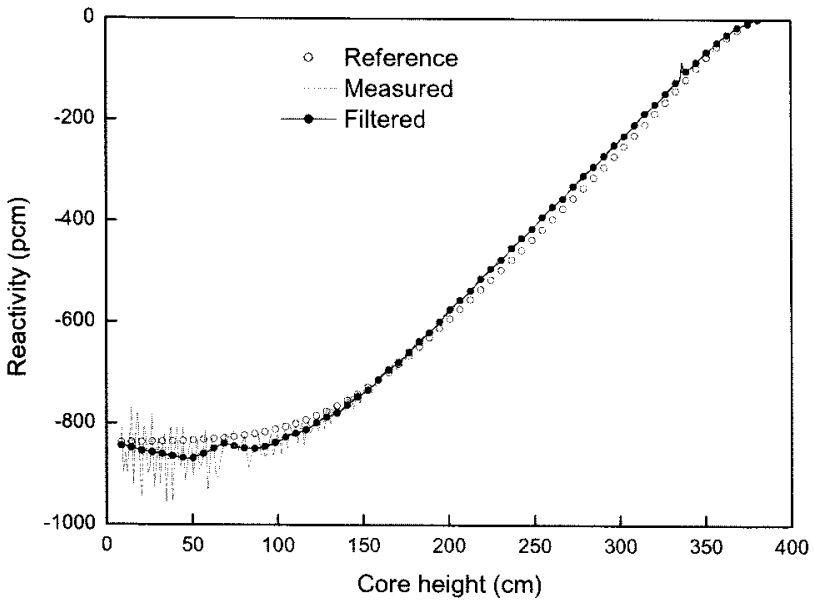

Fig. 5. Comparison of Calculated Control Bank Reactivity

\subsection{Edge-Preserving Kernel Filters}

The measurement signal of neutron density is normally noise-corrupted and control rod movement typically gives reactivity variation with edge signals like saw teeth. Those edge regions should be precisely preserved since the measured signal is used to estimate the reactivity worth which is a crucial parameter used to assure the safety of nuclear reactors.

In this section, the performance of three kinds of edge-preserving noise filters is demonstrated using simulated stepwise signals which are selected to emphasize the effect of signal edges. The tested filters are based on the unilateral with adaptive bandwidth, bilateral kernel and wavelet filters which are known to be effective in edge preservation.

Figure 6 shows the variation of reactivity signal and filtered signal of the prescribed noise filters. Figures 7

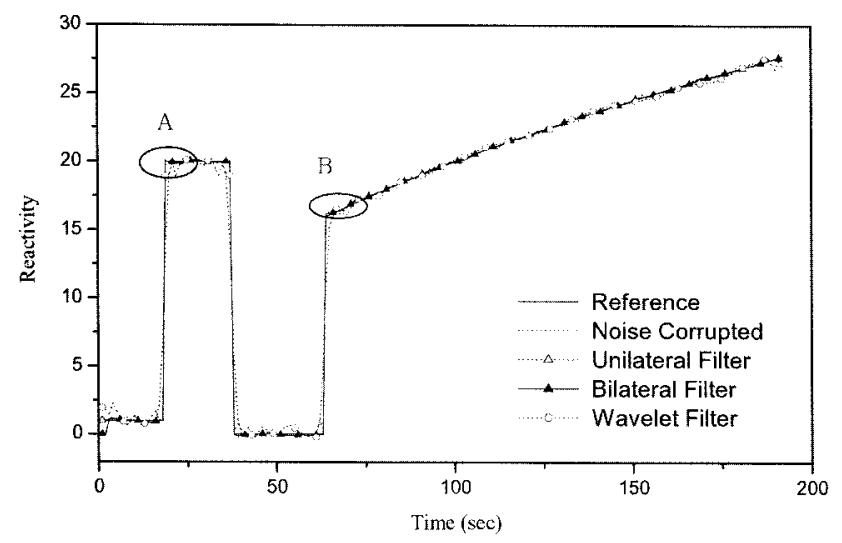

Fig. 6. Time-Varying Reactivity Signal with Edges and 8 show a magnified view of the filter performance at edge regions. The bilateral filter shows excellent edgepreserving properties compared with unilateral kernel filter with adaptive bandwidth or wavelet filters. This kind of stepwise variation of reactivity is frequently found during reactor startup physics test from movement of the control rods. Therefore the capability to reconstruct edge points is an important characteristic in a reactivity smoother.

Figure 9 shows the estimation error of the reactivity signal. The bilateral kernel filter gives a very small estimation error even at edge regions.

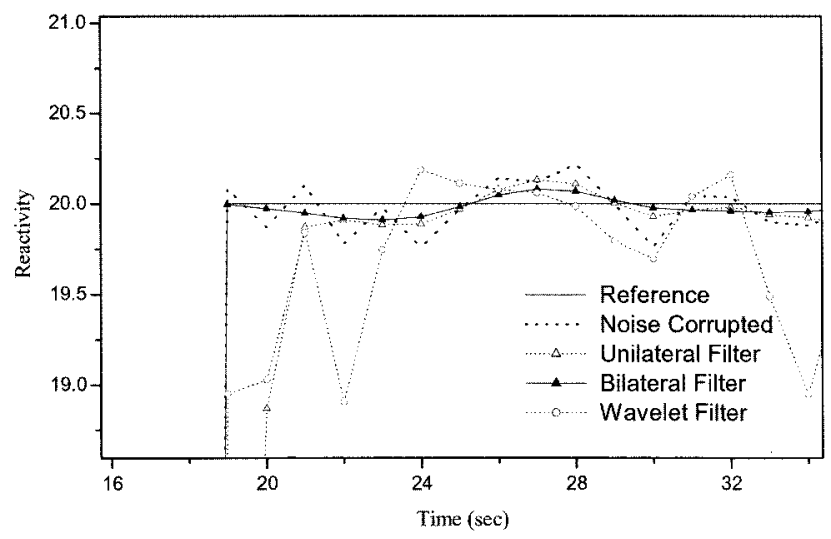

Fig. 7. Edge Signal at Region A

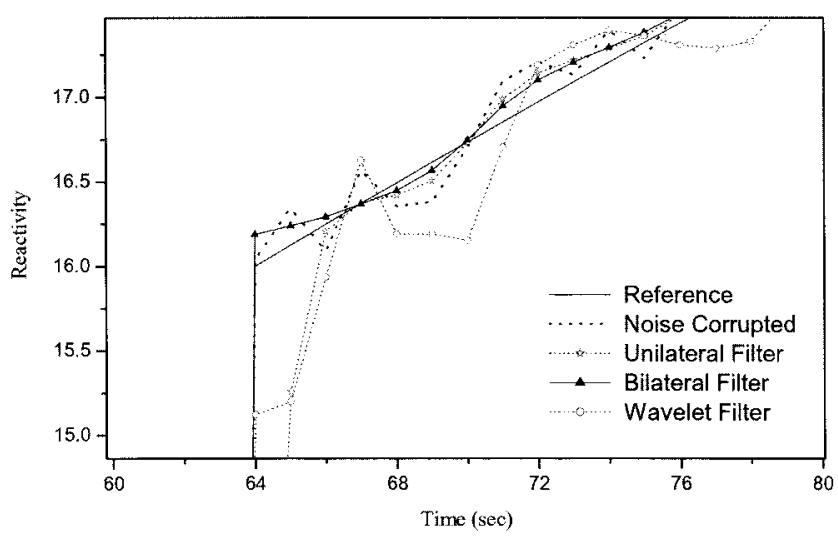

Fig. 8. Edge Signal at Region B

\section{CONCLUSION AND FURTHER WORKS}

The noise-filtering performance of kernel-based filters was demonstrated for reactivity measurements. A large-bandwidth unilateral kernel filter can be applied to 


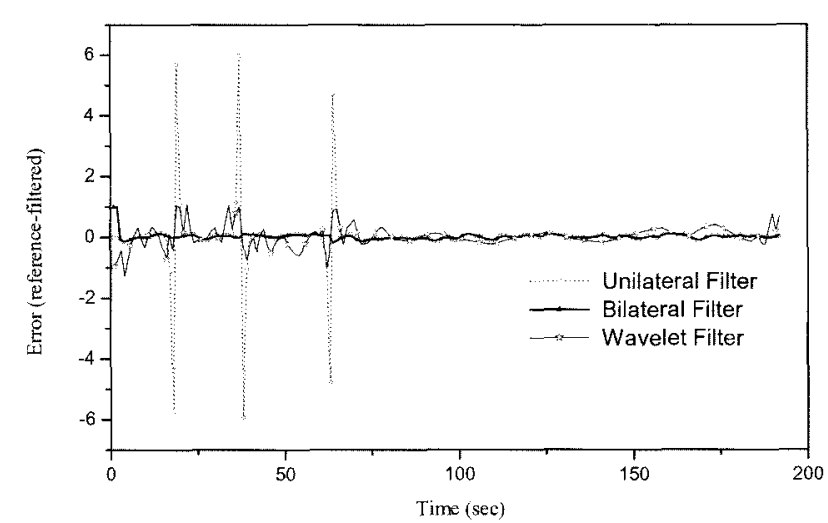

Fig. 9. Comparison of Estimation Error of the Reactivity Signal (Reference-Filtered)

the smooth but very weak neutron signal during dynamic control rod reactivity measurement in which the range of the reactivity variation is very large and the signal strength is below that of the background gamma levels. The bilateral filter shows a remarkable improvement in reconstructing an edged signal as compared with unilateral kernel and wavelet filters. Those filters have been developed and successfully applied in a digital reactivity computer system for reactor physics tests. The results show the developed filters can be applied not only for noise smoothing but also for bumpless following of a signal with non-smooth edges. Despite the enhanced filtering performance of the bilateral filter, it is desirable to find the robustness measure of the filter under low signal-to-noise ratio cases. A theoretical verification or extension of bilateral filter performance with reference to noise levels is therefore recommended for further work in this field.

\section{REFERENCES}

[1] Y. Shimazu, H. Unesaki and N. Suzuki, "Development of a compact digital reactivity meter and a reactor physics data processor," Nuclear Technology, 77, no. 3 (1987).

[2] E..K. Lee, H.C. Shin, S.M. Bae and Y.K. Lee., "New dynamic method to measure rod worths in zero power physics test at PWR startup," Annals of Nuclear Energy, 32, Issue 13, 2005.

[ 3 ] E. A. Narandaraya, "On estimating regression," Theory of Probability and its Application, 9, 1964.

[4] A. Moore, J. Schneider and K. Deng, "Efficient Locally Weighted Polynomial Regression Predictions," Proceedings of the Fourteenth International Conference on Machine Learning, 1997.

[ 5 ] M.G. Park, H. C. Shin, B. M. Koh and S. You et al., "Kernel Regression based Noise Smoothing of Reactivity Signal," Proceedings of the KNS Spring Meeting, 2005.

[6] C. Tomasi and R. Manduchi. "Bilateral filtering for gray and color images," Sixth International Conference on Computer Vision pp. 839-46. New Deli, India, 1998.

[7] M. Elad, "On the Origin of the Bilateral Filter and Ways to Improve It," IEEE Transactions on Image Processing, 11, no. $10,(2002)$

[8] A. Neumaier, "Wavelet Toolbox For Use with MATLAB" User's Guide", The MathWorks, Inc. (1996).

[9] M.G. Park, S. M. Bae and C. S. Lee., "Wavelet Filter Based De-Noising of Weak Neutron Flux Signal for Dynamic Control Rod Reactivity Measurement," Proceedings of the Korean Nuclear Society Autumn Meeting, Yongpyong, Korea, October 2002 